\title{
Efficiency and Equity in Collective Systems of Interacting Heterogeneous Agents
}

\author{
Akira Namatame, Saori Iwanaga \\ Dept. of Computer Science, National Defense Academy, Yokosuka, JAPAN \\ Email: nama@nda.ac.jp
}

\begin{abstract}
In this paper we address the issue realizing efficient and equitable utilization of limited resources by collective decision of interacting heterogeneous agents. There is no presumption that collective action of interacting agents leads to collectively satisfactory results without any central authority. How well agents do for it in adapting to their environment is not the same thing as how satisfactory an environment they collectively create. Agents normally react to others' decisions, and the resulting volatile collective decision is often far from being efficient. By means of experiments, we show that the overall performance crucially of the system on the types of interaction as well as the heterogeneity of preferences. We also show that the most crucial factor that considerably improves the performance is the way of information presentation to agents. It is shown that if each agent adapts to global information the performances are poor. The optimal guidance strategy to improve both efficiency and equity depends on the way of interaction. With symmetric interaction, the local information of the same type realizes the highest performance. With asymmetric interaction, however, the local information of the opposite preference type realizes the highest performance.
\end{abstract}

Keywords: interaction, heterogeneity, diversity, local adaptation, global adaptation, efficiency, equity

\section{Introduction}

The question of how it is possible for a group of independent individuals achieve both their own goals and a common goal has been addressed in many fields. By a common goal we mean a goal achievable by a group to require cooperation. The key element that distinguishes a common goal from an individual goal is that it requires collective action. Collective action, however, poses difficult problems, and it requires cooperation to overcome them. Coordination is different concept from cooperation, which does not assume the existence of the common goal shared by members. Coordination is necessary to achieve individuals' independent goals efficiently. The design of efficient collective action from bottom up becomes to a crucial issue in many disciplines [1][2]. It also an interesting problem is under what circumstances will a collection of interacting agents could realize efficient coordination from bottom up [11][12].

There are strong interests in many disciplines to answer the following questions: how do agents with heterogeneous micro-motives generate self-organized global macroscopic orders or regularities. However, there has been no natural methodology for systematically studying the dynamics of highly interacting heterogeneous agents. Some models treat adaptive processes with the assumption of the homogeneous payoff. Interdependent situations, in which an agent's decision depends on the decisions of other agents, are the ones that usually don't permit any simple summation or extrapolation to the aggregate. To make that connection we usually have to look at the system of collective interaction among individuals, which is also treated as the relation between individuals and the collectivity.

Collective means any pair of a complex system of autonomous components, together with a performance criterion by which we rank the behavior of the overall system. Wolpert and Tumer propose that the fundamental issue is to focus on improving our formal understanding of two 
closely related issues concerning collective [25][26]:

(1) The forward problem of how the fine-grained structure of the system underlying a collective determines its complex emergent behavior and therefore its performance.

(2) The inverse problem of how to design the structure of the system underlying a collective to induce optimal performance.

In examining collective, we shall draw heavily on the individual decisions. Indeed, an organization or society does not make decisions, individual do. It might be argued that understanding how individual make decisions is sufficient to understand and improve collective action. Much literature on collective has reacted against the older notion that irrationality is the key to explanation. They agree that collective often result from rational and calculated action. In this paper, we take a different view. Although individual decision is nested within important to understand, it is not sufficient to describe how a collection of agents arrives at specific decisions. These situations, in which an agent decision depends on the decisions of the others, are the ones that usually do not permit any simple summation or extrapolation to the aggregates. To make that connection we usually have to look at the system of interactions between agents and the collectivity. Sometimes the analysis of the collective is difficult, and it is inconclusive $[2][17][18][24]$. But even an inconclusive analysis warned against jumping to conclusions about the behavior of aggregates from what one knows or can guess about agent interests or motivations, or jumping to conclusions about agent intentions from the observations of aggregates.

We are also interested in how the society gropes for its way towards equilibrium in an imperfect world of locally interacting agents. There is no presumption that the self-interested behavior of agents should usually lead to collectively satisfactory results [9][14][20][21]. How well each agent does for it in adapting to its social environment is not the same thing as how satisfactory a social environment they collectively create for themselves. While all agents understand the outcome is inefficient, acting independently is powerless to manage this collective about what to do and also how to decide. The question of whether interacting heterogeneous agents self-organize efficient macroscopic orders from bottom up depends on how they interact each other. We attempt to probe deeper understanding this issue by specifying how they adapt each other. We consider two types of interaction, symmetric and asymmetric interaction. With symmetric interaction an agent receives a payoff if he chooses the same action as the majority does. With asymmetric interaction an agent receives a payoff if he chooses the same action as the minority does. Agents myopically adapt their behavior based on their idiosyncratic rule to others' behaviors. We analyze adaptive dynamics that relate the collective with the underlying individual adaptive behaviors. There are many parameters to be considered, among them, we examine the heterogeneity of utility and the configuration of locating agents. We evaluate emergent collective from the criteria of efficiency and equity. We show that interacting agents outperform when they adapt to local information rather than to global information.

\section{Formalism of the Model}

\subsection{Collectiveystems with micro-macro loop}

If the system consists of many interacting components, which we call agents, the system performance should be described on two different levels: the microscopic level, where the decisions of the individual agents occur and the macroscopic level where collective decision can be observed. To understand the role of a link between these two levels remains one of the 
challenges of complex system theory. Among many factors that may influence the performance of the overall system we focus on the information about the decisions of others available to each agent. The greatest promise lies in analysis of situations where agents behave in ways contingent on one another, and these situations are central in theoretical analysis of linking micro to macro levels of collective decision. We aim at discovering fundamental local or micro mechanisms that are sufficient to generate the macroscopic order of efficiency. This type of self-organization is often referred as collective orders emerged from the bottom up [3].

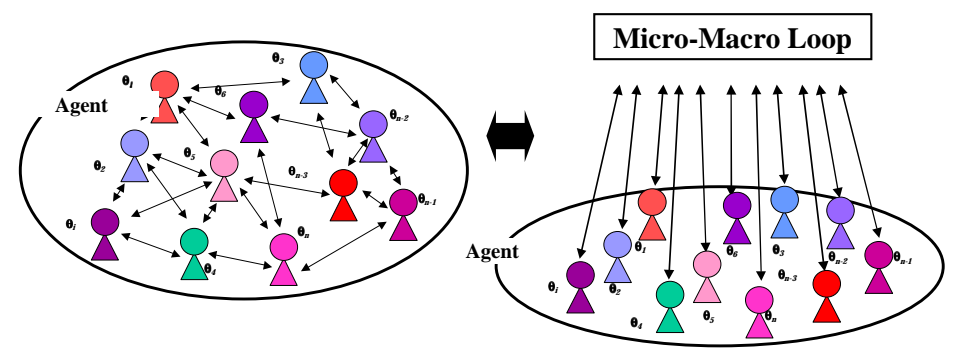

Fig.1: The systems of collective as micro-macro loop

\subsection{Examples of collective with interacting agents}

We address specific problems of collective with interacting agents. Social interactions pose many coordination problems to individuals. There are many situations where interacting agents can benefit from coordinating their action. Agents face problems of sharing and distributing limited resources in an efficient way.

\section{(1)Network formation by heterogeneous agents}

We consider a collection of agents $G=\left\{A_{i}: 1 \leq i \leq N\right\}$ in which each agent faces the binary decision problem. Agents periodically have to make decision to add the network or sever from it. Agents try to establish links in order to achieve higher payoffs. If the more agents join the network, the higher the payoff they receive, and this property is referred to as network externality [5][6]. Perhaps an essential point to put forward is that networks induce a special interdependency and a specific heterogeneity, which can affect network structure and aggregate phenomena in ways that are out of reach without them. To capture the intuition of this, it is enough to accept an agent's rational decision, depends on the agents it is directly linked with. This interdependence with heterogeneity in decision may in turn influence the evolution of the networks. Here, each agent $A_{i} \in G$ has the following two strategies:

$$
S_{1} \text { : Joins to the network, } \quad S_{2} \text { : Does not join the network }
$$

The number of agents to choose $S_{1}$ (trade) is denoted by $n(0 \leq n \leq N)$. Agent $A_{i}$ acquires a benefit of $a_{i}(n / N)$, a fixed value $a_{i}$ multiplied by the proportion of agents who join the network. If agent $A_{i}$ adds to the network, a cost $c_{i}$ is incurred. An agent can get a benefit as a spillover effect by $b_{i}(n / N) \quad\left(a_{i} \geq b_{i}\right)$, even if she does not add to the networks. The payoff of agent $A_{i}$ when she chooses $S_{1}$ or $S_{2}$ is given as follows:

$$
U_{i}\left(S_{1}\right)=a_{i}(n / N)-c_{i}, \quad U_{i}\left(S_{2}\right)=b_{i}(n / N)
$$

The utility of agent $A_{i}$ to choose $S_{1}$ or $S_{2}$ is illustrated as the function of $(n / N)$ in Fig.2. The utility of each agent is an increasing function of the population of agents in the network $n / N$. 


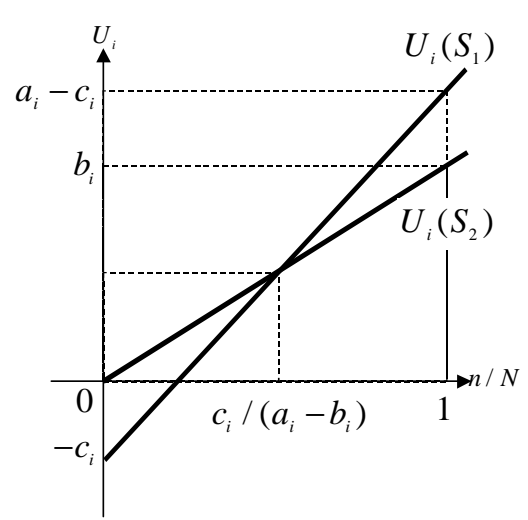

Fig.2: The utility of agent $A_{i}$ under $S_{1}$ or $S_{2}$ as the function of $(n / N)$

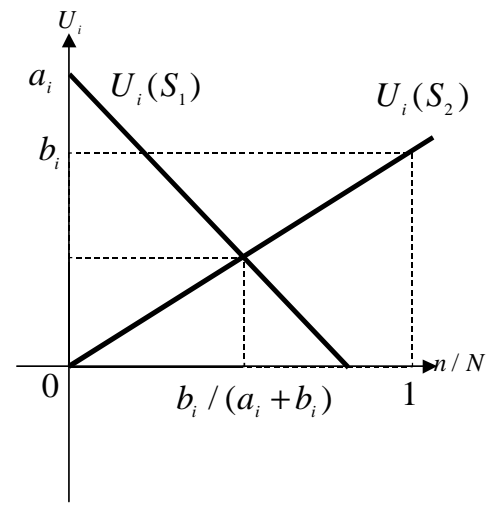

Fig.3: The utility of agent $A_{i}$ under $S_{1}$ or $S_{2}$ as the function of $(n / N)$

\section{(2)Route selection by heterogeneous agents}

There is another type of interaction in which we have to utilize different methodology. We consider a competitive routing problem of networks, in which the paths from sources to destination have to be established by independent agents. For example, in the context of traffic networks, agents have to determine their route independently. In telecommunication networks, they have to decide on what fraction of their traffic to send on each link of the network. Consider two parallel routes A and B, and each agent has to choose independently one of the two routes. As shown in Fig.3, each agent has the following two strategies:

$$
S_{1} \text { : Chooses the route A, } S_{2} \text { : Chooses the route B }
$$

The utility of each agent is determined what the majority does, and each agent gains utility only if she chooses the opposite route of the majority does. The utility function of agent $A_{i}$ if she chooses $S_{1}$ or $S_{2}$ is given as follows:

$$
U_{i}\left(S_{1}\right)=a_{i}(1-n / N), \quad U_{i}\left(S_{2}\right)=b_{i}(n / N)
$$

The payoff of agent $A_{i}$ if he chooses $S_{1}$ is a linearly decreasing function of the proportion of the same decision. On the other hand, the payoff if he chooses $S_{2}$ is a linearly increasing function of the proportion of agents with the opposite decision. The utility of agent $A_{i}$ when she chooses $S_{1}$ or $S_{2}$ is illustrated s as the function of $(n / N)$ in Fig.4.

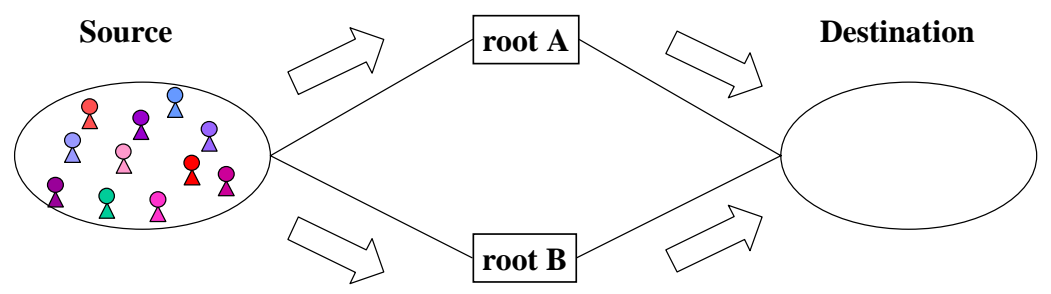

Fig.4 A competitive route selection

Large-scale interactions with $\mathrm{N}$-persons can be decomposed into the problem of the interaction between an individual and the aggregate. In the network formation problem in the previous section, we define the payoff matrix of each agent as given in Table 1. 
Table 1 The payoff matrix of agent $A_{i}$

\begin{tabular}{|c|c|c|}
\hline Choice of agent $A_{i}$ & $S_{1}$ & $S_{2}$ \\
\hline$S_{1}$ & $a_{i}-c_{i}$ & $-c_{i}$ \\
\hline$S_{2}$ & $b_{i}$ & 0 \\
\hline
\end{tabular}

Table 2 The payoff matrix of agent $A_{i} \quad\left(0 \leq \theta_{i} \leq 1\right)$

\begin{tabular}{|c|c|c|}
\hline $\begin{array}{r}\text { Choice of other } \\
\text { Agents } \\
\text { Choice of agent } A_{i}\end{array}$ & $S_{1}$ & $S_{2}$ \\
\hline$S_{1}$ & $1-\theta_{i}$ & 0 \\
\hline$S_{2}$ & 0 & $\theta_{i}$ \\
\hline
\end{tabular}

The expected payoff of agent $A_{i}$ if she chooses $S_{1}$ or $S_{2}$ is given as follows:

$$
U_{i}\left(S_{1}\right)=\left(a_{i}-c_{i}\right)(n / N)-c_{i}(1-n / N)=a_{i}(n / N)-c_{i}, \quad U_{i}\left(S_{2}\right)=b_{i}(n / N)
$$

Therefore, we have the same utility function with (2.2). Since matrix of the two-person game is invariant for the affine transformation [27], the payoff matrix of Table 1 can be equivalently transformed into the matrix of Table 2, where $\theta_{i}=c_{i} /\left(a_{i}-b_{i}\right)$.

Similarly the payoff matrix of agent $A_{i}$ in the route selection problem can be given as in Table 3. The matrix of Table 3 can be also equivalently transformed into the matrix of Table 4 , where $\theta_{i}=b_{i} /\left(a_{i}+b_{i}\right)$.

Table 3 The payoff matrix of agent $A_{i} \quad$ Table 4 The payoff matrix of agent $A_{i} \quad\left(0 \leq \theta_{i} \leq 1\right)$

\begin{tabular}{|c|c|c|}
\hline $\begin{array}{r}\text { Choice of other } \\
\text { Agents } \\
\text { Choice of agent } A_{i}\end{array}$ & $S_{1}$ & $S_{2}$ \\
\hline$S_{1}$ & 0 & $a_{i}$ \\
\hline$S_{2}$ & $b_{i}$ & 0 \\
\hline
\end{tabular}

\begin{tabular}{|c|c|c|}
$\begin{array}{r}\text { Choice of other } \\
\text { Agents }\end{array}$ & $S_{1}$ & $S_{2}$ \\
Choice of agent $A_{i}$ & & \\
\hline$S_{1}$ & 0 & $\theta_{i}$ \\
\hline$S_{2}$ & $1-\theta_{i}$ & 0 \\
\hline
\end{tabular}

They have to coordinate their decisions with others in order to improve their utility. We distinguish the two types of the above interaction as symmetric and asymmetric interaction [18][19]. Interaction usually implies that increased effort by some agents leads the remaining agents to follow suit, which gives multiplier effects [16]. We call this type of interaction as symmetric interaction. On the other hand, in the route selection problem, agents receive payoff if they select the opposite strategy as the majority does. This type of interaction is distinguished as asymmetric interaction. This type interaction is also referred as minority games in which agents receive payoff if he selects the same strategy as the minority does [1][19][23]. For realizing efficient and equitable distributions of limited resources, agents normally react to aggregate of others' decisions. The resulting volatile collective decision is often far from being efficient. The overall performance depends crucially on the type of interaction as well as the heterogeneity of agent preferences.

\section{Global Adaptation vs. Local Adaptation}

In examining collective decisions, we shall draw heavily on the individual adaptive decisions. Within the scope of our model, we treat models in which agents make deliberate decisions by applying rational procedures, which also guide their reasoning. In order to describe the adaptation process at the individual level, we may have two fundamental models, global adaptation and local adaptation. It is important to consider with whom an agent interacts and how each agent decides his action depending on others' actions. Agents may adapt based on the aggregate information representing the current status of the whole system (global adaptation) as shown in Fig.5 (a). In this case, each agent chooses an optimal decision based on aggregate information about how all other agents behaved in the past. An agent calculates her reward and 
plays her best response strategy. An important assumption of global adaptation is that they receive knowledge of the aggregate.

In many situations, agents are not assumed to be knowledgeable as to correctly guess or anticipate other agents' actions, or they are less sophisticated and that they do not know how to calculate best replies [11]. With local adaptation each agent is modeled to adapt to her neighbors [10][14]. The hypothesis of local adaptation also reflects limited ability of agents' parts to receive, decide, and act based upon information they receive in the course of interaction. As a specific model, we consider the lattice structure as shown in Fig.5 (b), in which each agent interacts with his neighbors. The main point is that an agent's decision depends on what it knows about others. At each period of time, each agent decides whether to add or sever the network given the knowledge. An agent thinks strategically, knowing that everyone else is also making a rational decision given global or local information.

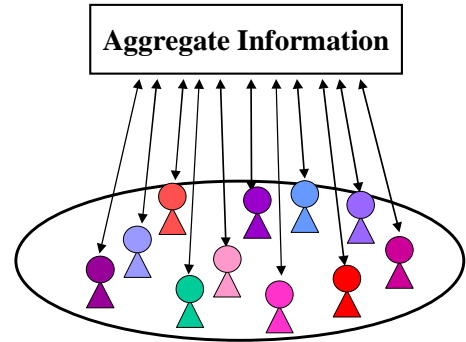

(a) Global adaptation

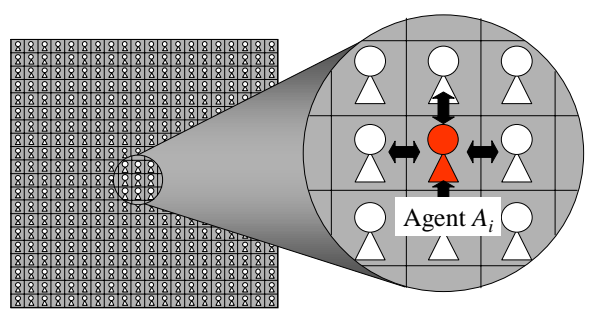

(b) Local adaptation

Fig.5: Two basic adaptation models of agents

\section{[Global adaptation and symmetric interaction]}

We obtain the adaptive rule of each agent as her best response. Let's denote the proportion of agents having chosen $S_{1}$ at time $t$ in a population by $p(t)$. Agent $A_{i}$ of the payoff parameter $\theta_{i}$ in Table 2 calculates her expected utilities as follows:

$$
\bar{U}_{i}\left(S_{1}\right)=p(t)\left(1-\theta_{i}\right), \quad \bar{U}_{i}\left(S_{2}\right)=(1-p(t)) \theta_{i}
$$

By comparing the expected utilities under $S_{1}$ and $S_{2}$, the optimal adaptive rule of agent $A_{i}$ is obtained as the function of the aggregate information on collective $p(t)$ and her idiosyncratic payoff $\theta_{i}$ (defined as threshold) as follows:

(i) If $p(t) \geq \theta_{i}$, choose $S_{1}$

(ii) If $p(t)<\theta_{i}$, choose $S_{2}$

\section{[Local adaptation and symmetric interaction]}

The local adaptive rule is obtained as follows. Let's denote the proportion of the neighbors of agent $A_{i}$ having chosen $S_{1}$ at time $t$ by $p_{i}(t)$. The optimal adaptive rule with local adaptation is obtained as follows:

(i) If $p_{i}(t) \geq \theta_{i}$, choose $S_{1}$

(ii) If $p_{i}(t)<\theta_{i}$, choose $S_{2}$

\section{[Global adaptation and asymmetric interaction]}

We obtain the adaptive rules of agents with asymmetric interaction. Let's denote the proportion of agents having chosen $S_{1}$ at time $t$ in a population by $p(t)$. Agent $A_{i}$ of the payoff parameter $\theta_{i}$ in Table 3 calculates her expected utilities as follows: 


$$
\bar{U}_{i}\left(S_{1}\right)=(1-p(t)) \theta_{i}, \quad \bar{U}_{i}\left(S_{2}\right)=p(t)\left(1-\theta_{i}\right)
$$

By comparing the expected utilities under $S_{1}$ and $S_{2}$, the optimal adaptive rule of agent $A_{i}$ is obtained as the function of the aggregate information on collective $p(t)$ and her idiosyncratic threshold $\theta_{i}$ as follows:

(i) If $p(t) \leq \theta_{i}$, choose $S_{1}$

(ii) If $p(t)>\theta_{i}$, choose $S_{2}$

\section{[Local adaptation and asymmetric interaction]}

The adaptive rule with local adaptation is obtained as follows: Let's denote the proportion of the neighbors of agent $A_{i}$ who have chosen $S_{1}$ at time $t$ by $p_{i}(t)$. The optimal adaptive rule with local adaptation is obtained as follows:

(i) If $p_{i}(t) \leq \theta_{i}$, choose $S_{1}$

(ii) If $p_{i}(t)>\theta_{i}$, choose $S_{2}$

\section{Heterogeneity in Preferences and Representation of Diversity}

The crucial concept for describing heterogeneity of agents is their preference characterized by their threshold $\theta_{i}$. Threshold models choose the elements of collective which game theory handles only with difficulty and makes them central: substantial heterogeneity of preferences and inter dependence of decisions over time. This is possible because that the payoff matrix is replaced by a one-dimensional vector of threshold one for each agent, which allows enormous simplification in the ensuring analysis.

In this section, we consider several collections of heterogeneous agents. We also characterize the diversity of heterogeneous agents from both threshold distribution and the configuration of their location. Each agent has an idiosyncratic threshold $\theta_{i}$, and the diversity of heterogeneous agents is represented by the distribution of $\theta$. We classify heterogeneous agents into the following two types:

(1) Type 1: Agent with the threshold $\theta_{i} \leq 0.5$. (Such an agent prefers the strategy $S_{1}$ to $S_{2}$.)

(2) Type 2: Agent with the threshold $\theta_{i}>0.5$. (Such an agent prefers the strategy $S_{2}$ to $S_{1}$.)

We also classify interaction types as follows:

(1)Random neighbor: Each agent interacts with neighbors of any type.

(2)Homogeneous neighbor: Each agent interacts with neighbors of the same type.

(3)Heterogeneous neighbor: Each agent interacts with neighbors of the opposite type.

We assume the proportions of agents of Type 1 and Type 2 in a collection of agents $G=\left\{A_{i}: 1 \leq i \leq N\right\}$ are the same. We denote the number of agents with the same threshold $\theta$ in $G$ by $n(\theta)$. We define the density of $\theta$ by $f(\theta)$, which is obtained by divided $n(\theta)$ by the total number of agent $N$,

$$
f(\theta)=n(\theta) / N .
$$

We also assume that the threshold distribution $f(\theta)$ is symmetric with the property

$$
f(\theta)=f(1-\theta) .
$$

As specific examples, we consider the distribution functions as shown in Fig.6. In these cases, the average of the threshold is 0.5 , i.e.,

T $f(\theta) d \theta=0.5$. 


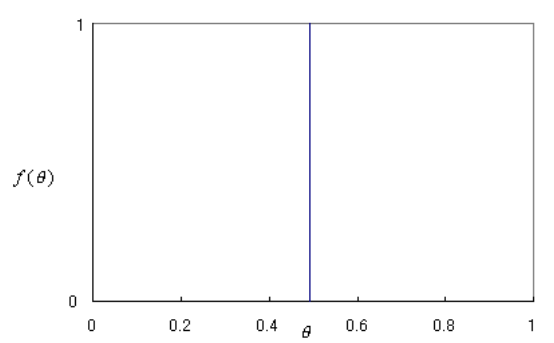

(a) Case 1 (Identical distribution)

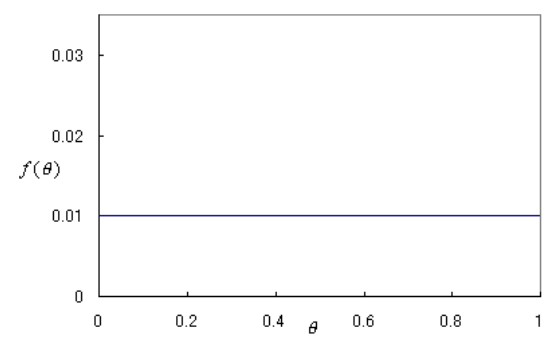

(c) Case 3 (Uniform distribution)

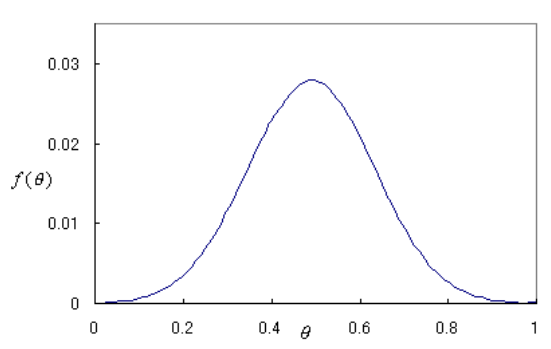

(b) Case 2 (Normal distribution)

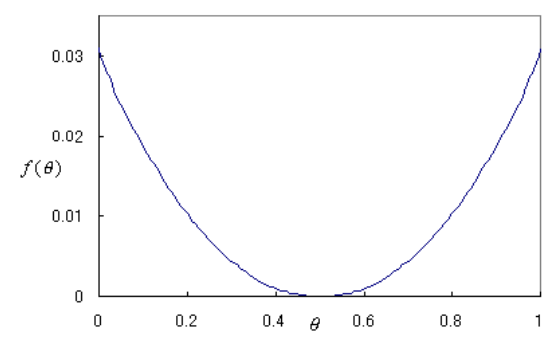

(d) Case 4 (Polarized distribution)

Fig.6: Several distribution functions of threshold: $f(\theta)$
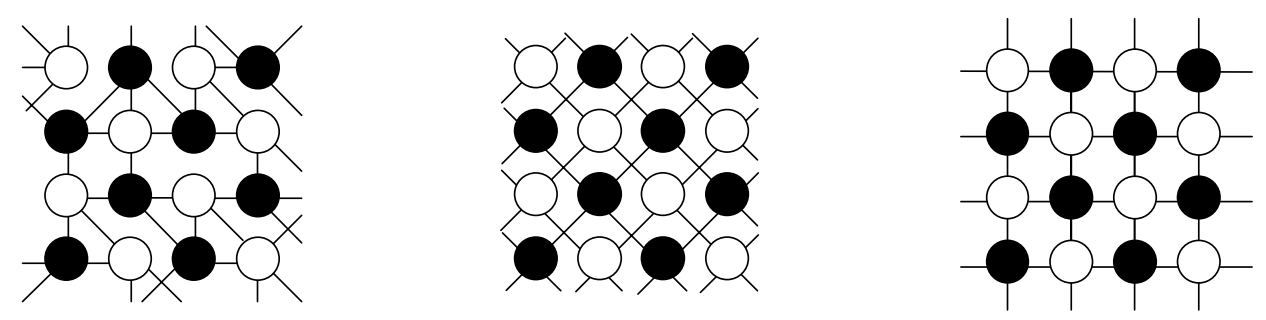

OType1: An agent who prefer $S_{1}$ to $S_{2}$ OType2: An agent who prefer $S_{2}$ to $S_{1}$

$\begin{array}{llll}\text { (a) Random neighbor } & \text { (b) Homogeneous neighbor } & \text { (c) Heterogeneous neighbor }\end{array}$ Fig.7: Configurations of neighbor

\section{Evaluation of the Systems of Collective}

In this section, we evaluate collective of interacting heterogeneous agents with three criteria, stability, efficiency and equity. The stability of collective is from the point of the path-dependency of collective. We obtain and evaluate the proportion of agents who choose each strategy staring from any initial condition. Efficiency is evaluated by obtaining the average utility, which also stands for the measure of the desirability at the macro level. Equity is evaluated by obtaining the utility distribution, which stand for the measure of the desirability at the micro level.

\section{(1) Collective under symmetric interaction} $<$ Efficiency >

We characterize collective at equilibrium by $p^{*}$, which represents the proportion of agents to choose $S_{1}$ at equilibrium. The efficiency of global adaptation is evaluated as follows: The adaptive rule of each agent at equilibrium is given by (3.2). Agents with the threshold $\theta$ is less than or equal to $p^{*}$ receive the utility at the level of

$$
u=p^{*}(1-\theta) \quad\left(0 \leq \theta \leq p^{*}\right) \text {. }
$$


Agents whose threshold $\theta$ are greater than $p^{*}$ choose $S_{2}$, and then they receive their utility at the level of:

$$
u=\left(1-p^{*}\right) \theta \quad\left(p^{*}<\theta \leq 1\right)
$$

Therefore, the average utility $\bar{U}$ is obtained as:

$$
\bar{U}=\mathbb{Z}(1-\theta) p^{*} f(\theta) d \theta+\mathbb{Z} \theta\left(1-p^{*}\right) f(\theta) d \theta
$$

From this equation, the average payoff (efficiency) $\bar{U}$ depends on collective at equilibrium $p^{*}$ and the threshold distribution $f(\theta)$.

We consider of the following typical cases:

(i) All agents choose the same strategy: $S_{1}\left(p^{*}=1\right)\left(\right.$ or $\left.S_{2}\left(p^{*}=0\right)\right)$.

(ii) A half of agents choose $S_{1}$ and the rest of agents choose $S_{2}\left(p^{*}=0.5\right)$.

Efficiencies of the above two cases are obtained as,

(i) $p^{*}=1\left(\right.$ or $\left.p^{*}=0\right): \bar{U}=0.5$

(ii) $p^{*}=0.5: \bar{U}=0.5-\mathbb{Z} \theta f(\theta) d \theta$

\section{$<$ Equity >}

The proportion of agents who gain the utility $u$ by choosing $S_{1}$ is given as $f(1-\theta)$. Therefore, we have the utility distribution as follows:

$$
g_{1}(u)=f(1-\theta)=f\left(u / p^{*}\right) \quad\left(0 \leq \theta_{i} \leq p^{*}, p^{*} \neq 0\right)
$$

The proportion of agents who gain the utility $u$ by choosing $S_{2}$ is given by $f(\theta)$. Therefore, we have the utility distribution.

$$
g_{2}(u)=f(\theta)=f\left(u /\left(1-p^{*}\right)\right) \quad\left(p^{*}<\theta_{i} \leq 1, p^{*} \neq 1\right)
$$

Therefore, the overall utility distribution is obtained as:

$$
g(u)=g_{1}(u)+g_{2}(u) .
$$

The utility distribution $g(u)$ of collective equilibrium $p^{*}$ is obtained as:

(i) $p^{*}=1 \quad\left(\right.$ or $\left.p^{*}=0\right): g(u)=f(u) \quad(0 \leq u \leq 1)$

(ii) $\quad p^{*}=0.5: \quad g(u)=2 f(2 u)(0.25 \leq u \leq 0.5)$

The Gini ratio $\varphi(0 \leq \varphi \leq 1)$ is often used to measure the extent to which the utility distribution of a society. The Gini ratio can be obtained from a Lorenz curve. The Gini ratio is obtained by measuring the area surrounded by the Lorenz curve $L(x)$ in Fig.8. The $x$-axis represents the cumulative proportion of agents and $y$-axis represents the cumulative proportion of the total utility $L(x)$, which is cumulated to the proportion at the level $x$ starting with the poorest agents. The Gini ratio $\varphi$ is then defined as

$$
\varphi=1-2 \mathbb{Z}(x) d x .
$$

The measure of equity $E$ which global adaptation is then obtained as follows: 


$$
E=1-\varphi=2 \mathbb{Z}(x) d x
$$

Using the proportion of agents $g(u)$ who obtain the utility level $u$, the Lorenz curve $L(x)$ is given as follows:

$$
L(x)=\mathbb{Z} \tau g(\tau) d \tau / \mathbb{Z}_{g}(\tau) d \tau
$$

Where $w$ is defined as the parameter satisfying $x=\mathbb{Z} g(\tau) d \tau$.

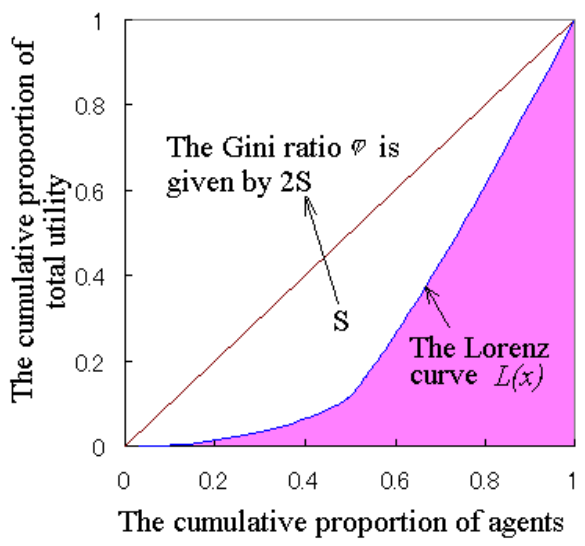

Fig.8: The Lorenz curve and the Gini ratio

\section{(2) Collective under asymmetric interaction}

The adaptive rule of an agent who faces asymmetric interaction is given by (3.5). Agents whose threshold $\theta$ is less than equal $p^{*}$ at equilibrium receive the utility at the level of

$$
u=\left(1-p^{*}\right) \theta \quad\left(p^{*} \leq \theta \leq 1\right)
$$

Agents whose threshold $\theta$ is greater than $p^{*}$ choose $S_{2}$, and they receives utility

$$
u=p^{*}(1-\theta) \quad\left(0<\theta \leq p^{*}\right) .
$$

The average utility $\bar{U}$ (efficiency) is obtained as the same as given in (5.3), and the efficiency and equity are the same as the case of symmetric interaction.

\section{Simulation Results}

We arrange a collection of heterogeneous agents in the area of $50 \times 50$ (2500 agents in total) with no gap, and four corners and an edge of an area connect it with an opposite side. The consequence of their actions also gives an effect on agents with whom not directly linked. We are interested in the long-run behavior of collective when heterogeneous interacting agents adapt their decisions over time. We especially investigate how agents' decisions combine with the decisions of the others produces the macro behavior. We impose only a weak monotonic condition reflecting the inertia and myopia hypotheses on the dynamics, which describe the changes in the number of agents playing each strategy.

\subsection{Collective of Symmetric Interaction}

\section{(1) Global adaptation}

The simulation results of global adaptation are shown in Fig.9. In Fig.9 (a), the $x$-axis represents the proportion of agents having chosen $S_{1}$ initially and the $y$-axis represents 
collective $p^{*}$ at equilibrium. Collective decision $p^{*}$ at equilibrium depends on both the population structure and the initial value. In Fig.9 (b), and the $y$-axis represents the average utility $\bar{U}$ at equilibrium. In all cases, efficiencies $\bar{U}$ are less than 0.5. In Fig.9 (c), the $y$-axis represents the equity $E$ at equilibrium.

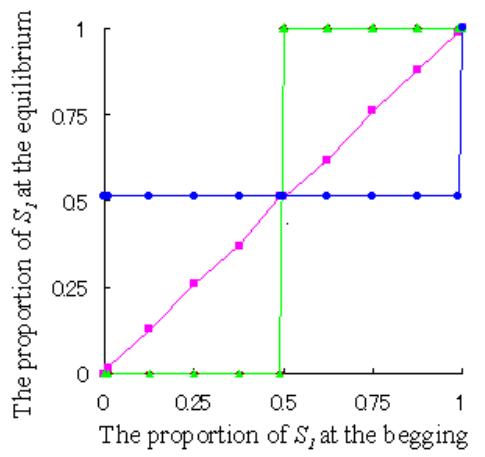

(a) The collective

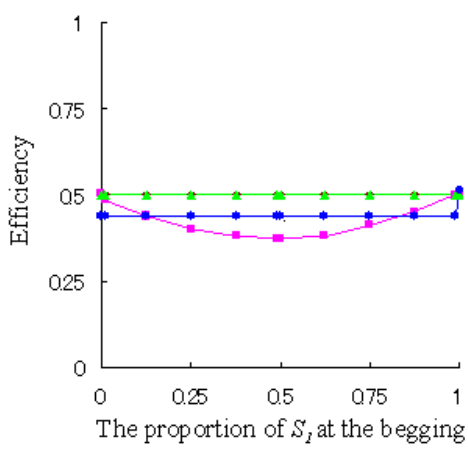

(b) The efficiency $\triangle$ case 2 case3

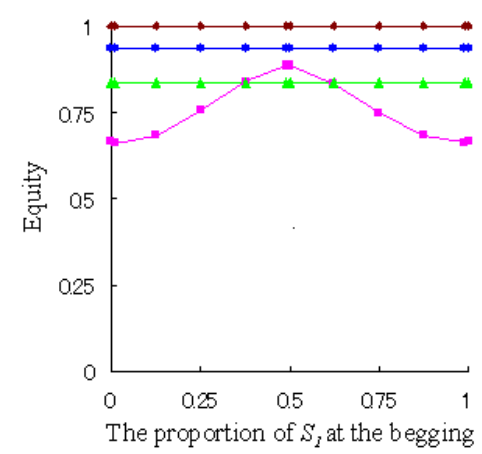

(c) The equity

Fig.9: Simulation results of global adaptation

\section{(2)Local adaptation to random neighbors}

The simulation results of local adaptation with random neighbors are shown in Fig.10. Collective behavior is similar to the global adaptation model in Fig.9 (a), although the turning point is dull in all cases as shown in Fig.10 (a). The average utilities $\bar{U}$ of all cases are almost 0.5 (Fig.10 (b)). Efficiency is also close to the case of the global adaptation (Fig.9 (b)).

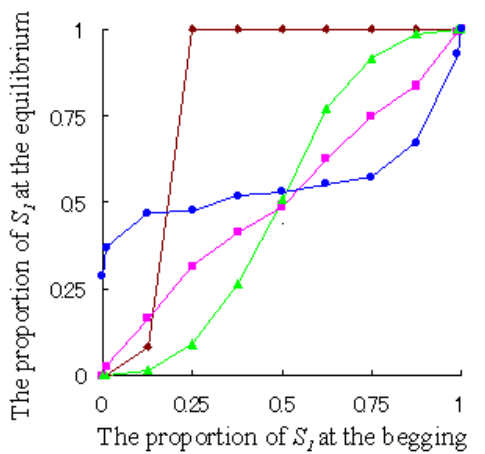

(a) The collective

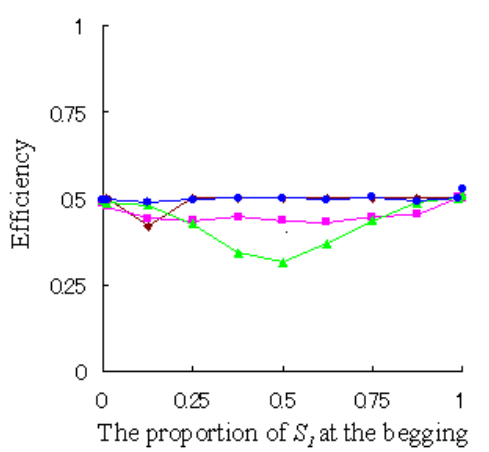

(b) The efficiency

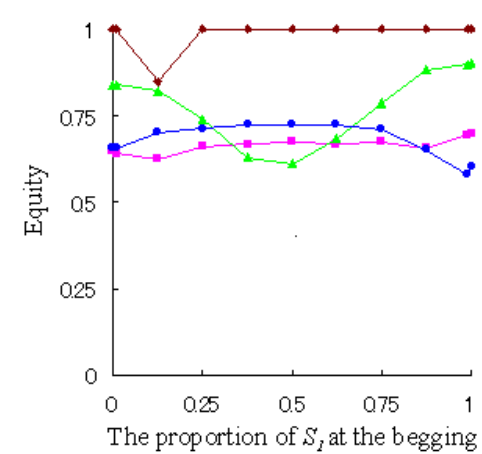

(c) The equity

Fig.10: Simulation results of local adaptation

\section{(3) Local adaptation to homogeneous neighbors}

The simulation results with homogeneous neighbors are shown in Fig.11. Collective behavior $p^{*}$ at equilibrium is about 0.5 starting from any initial point in Case 2, 3 and 4 (Fig.11 (a)). Simulation result of Case 1 is the same as the adaptation to random neighbors. The average utilities $\bar{U}$ of all cases become to be more than 0.5 (Fig.11 (b)) and equity $E$ are also high (Fig.11 (c)). From these results, local adaptation to homogeneous neighbors result in desirable behavior. 


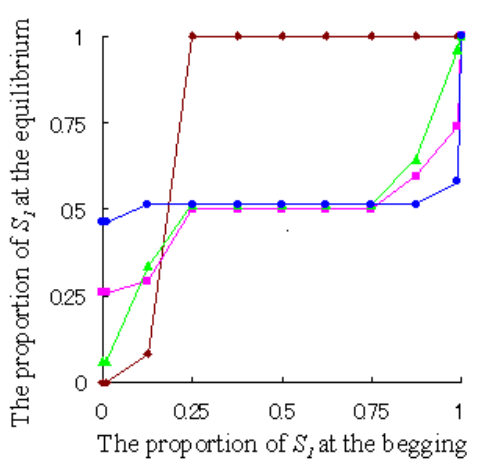

(a) The collective

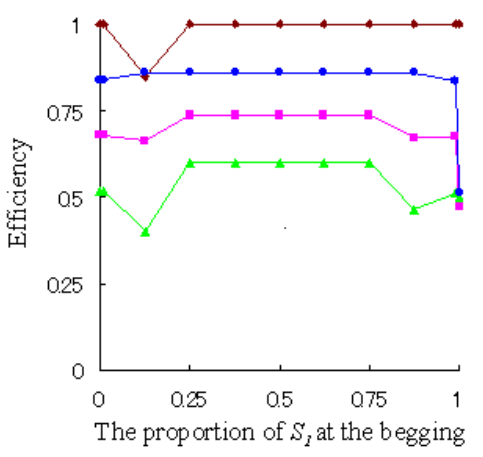

(b) The efficiency $\triangle$ case 2 case 3

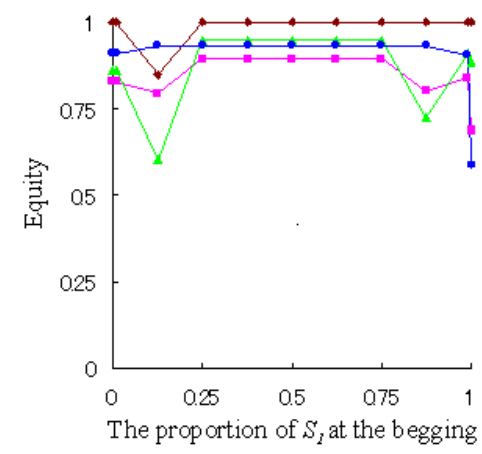

(c) The equity

case 4

Fig.11: Simulation results of local adaptation

\section{(4)Efficiency and equity}

In Fig.12, we summarize the simulation results. The $x$-axis represents equity $E$ and the $y$-axis represents efficiency, in term of the average utility $\bar{U}$. With the global adaptation model, we obtain as follows: Case $1:(E, \bar{U})=(1.0,0.5)$, Case $2:(0.84,0.5)$, Case $3:(0.89,0.38)$ and Case $4:(0.94,0.44)$. For the local adaptation model with random neighbors, we obtain as follows: Case 1: $(E, \bar{U})=(1.0,0.5)$, Case $2:(0.61,0.31)$, Case $3:(0.67,0.44)$ and Case $4:(0.73$, $0.50)$. With the local adaptation model with homogeneous neighbors, we obtain as follows: Case 1: $(E, \bar{U})=(1.0,0.5)$, Case 2: $(0.95,0.6)$, Case 3: $(0.89,0.73)$ and Case $4:(0.93,0.86)$. Efficiency and equity are different depending on the way of interaction, although collective are almost the same.

For a population of identical agents (Case 1), all agents have the same payoff and equity is the highest, however, the efficiency is moderate. In global adaptation with divers population, efficiency is moderate and equity is also high. In local adaptation with random neighbors, both efficiency and equity are low, and diversity tightens gap in efficiency and equity. with the homogeneous neighbors, both efficiency and equity become high.

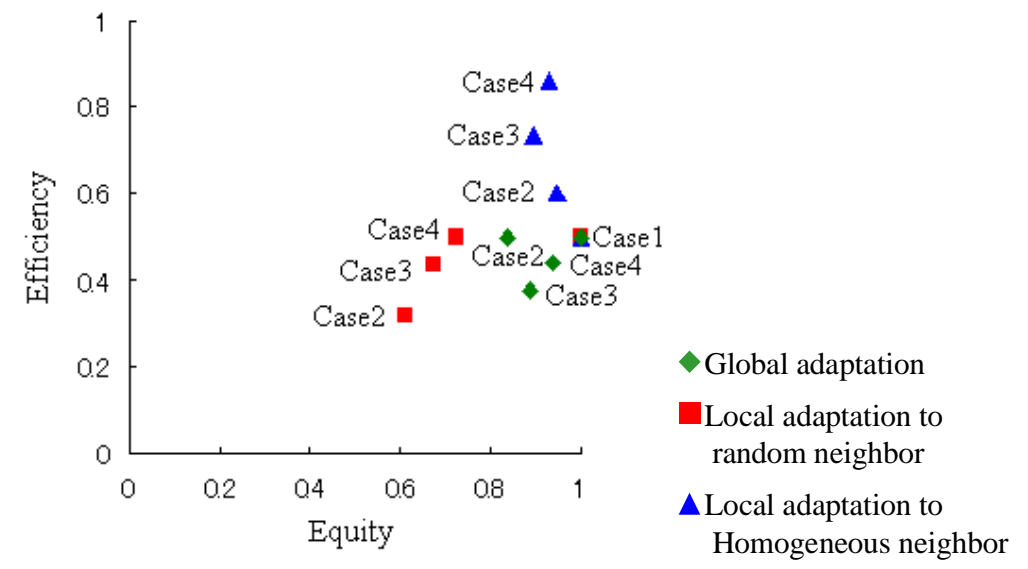

Fig.12: Efficiency and equity (the initial proportion: 0.5 )

\subsection{Collective of Asymmetric Interactions}

\section{(1) Global adaptation}

The simulation results of global adaptation are shown in Fig.13 since oscillation appears, with asymmetric interaction, we describe both maximum and minimum value of $p(t)$ in Fig.13 (a). 
However, in Case 3 and 4, oscillations are suppressed and efficiency $\bar{U}$ and equity $E$ become high.

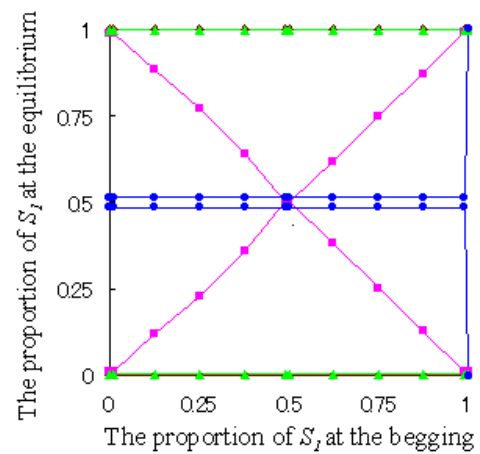

(a) The collective

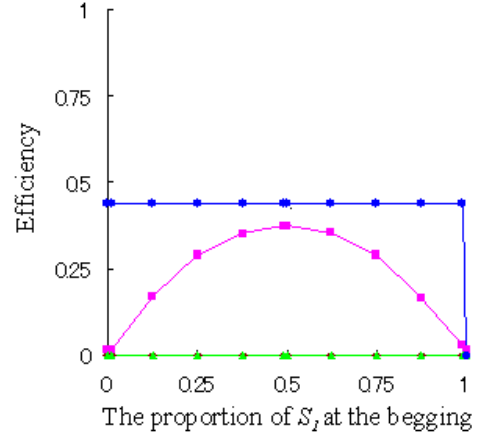

(b) The efficiencv case $1-$ case $2-$ case 3

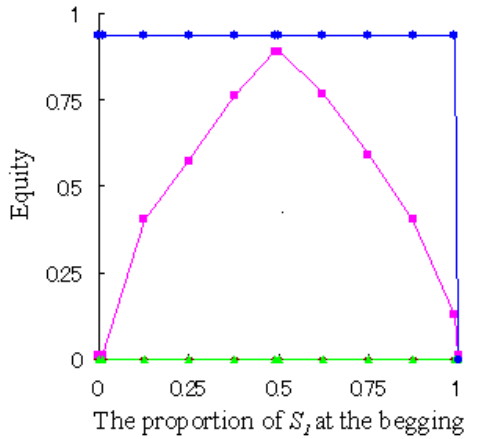

(c) The equity

Fig.13: Simulation results of global adaptation

\section{(2)Local adaptation to random neighbors}

The simulation results of local adaptation to random neighbors are shown in Fig.14. Collective behavior is similar to the global adaptation model as shown in Fig.13 (a), although the turning point is dull in all cases. In any cases, there are huge discrepancies among agents, the average utilities $\bar{U}$ are less 0.5 and equities $E$ are low. From these results, the local adaptation model with random neighbors is becomes the same as the global adaptation model.

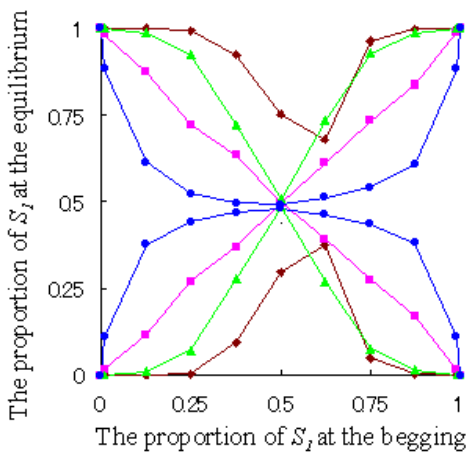

(a) The collective

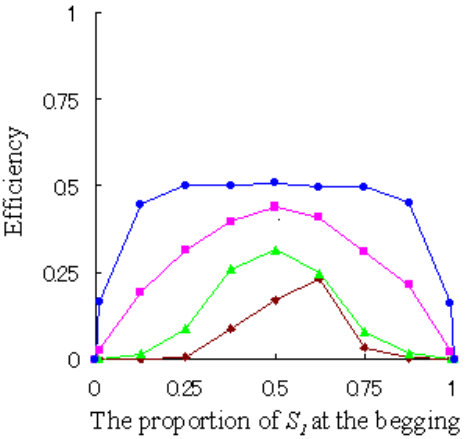

(b) The efficiency case $1-$ case $2-$ case 3

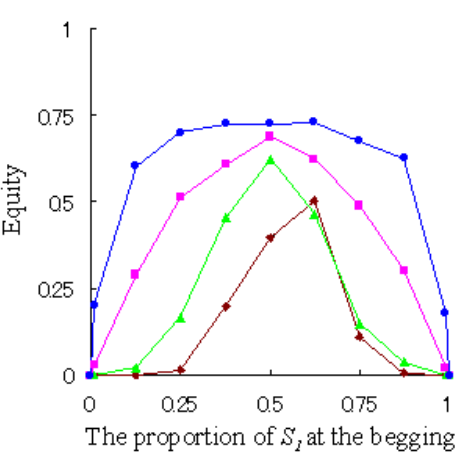

(c) The equity

Fig.14: Simulation results of local adaptation

\section{(3) Local adaptation to heterogeneous neighbors}

The simulation results of local adaptation to homogeneous neighbors are shown in Fig.15. The collective behavior $p^{*}$ at equilibrium is obtained as about 0.5 starting from any initial condition in Case 2, 3, and 4 as shown in Fig.15 (a). In spite of diversity of preferences, they show same as collective with the local adaptation to homogeneous neighbors. From these results, homogeneous interaction gives rise to the most desirable collective behavior by overcoming diversity of preferences. 


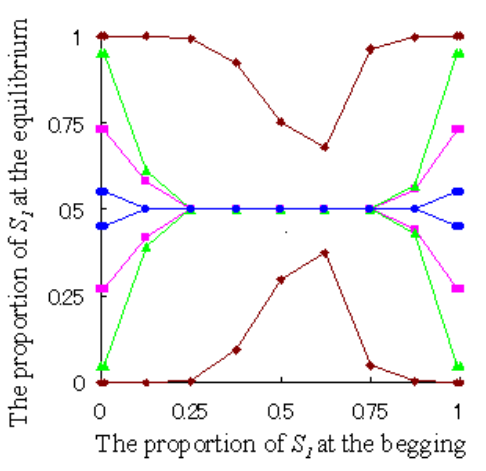

(a) The collective

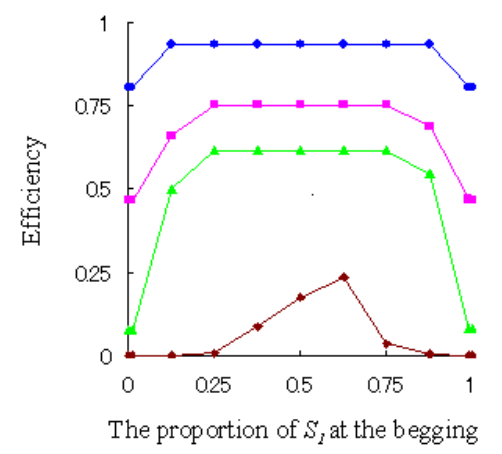

(b) The efficiency

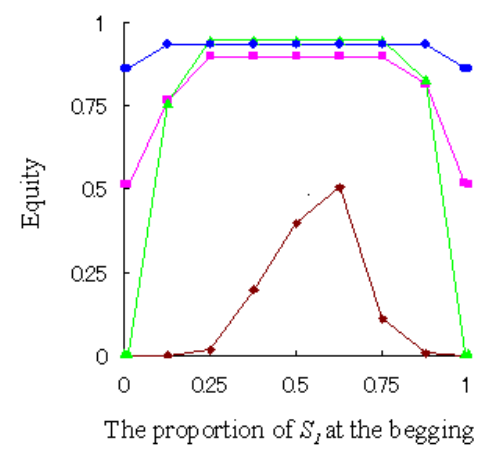

(c) The equity case $1 \Delta$ case 2 case3

Fig.15: Simulation results of local adaptation with heterogeneous neighbors

\section{(4)Efficiency and equity}

In Fig.16, we summarize the simulation results in terms of equity and efficiency. The $x$-axis represents equity $E$ and the $y$-axis represents efficiency, the average utility $\bar{U}$. For the global adaptation model, we obtain as: Case 1: $(E, \bar{U})=(0.0,0.0)$, Case 2: $(0.0,0.0)$, Case 3: $(0.89,0.38)$ and Case $4:(0.94,0.44)$. For the local adaptation model to random neighbors, we obtain as: Case 1: $(E, \bar{U})=(0.69,0.17)$, Case 2: $(0.56,0.31)$, Case 3: $(0.66,0.44)$ and Case 4: $(0.73,0.51)$. For the Local adaptation model with homogeneous neighbors, we obtain as: Case $1:(E, \bar{U})=(0.69,0.17)$, Case $2:(0.73,0.51)$, Case $3:(0.95,0.75)$ and Case $4:(0.93,0.87)$.

For a collection of identical agents (Case 1), almost agents choose the same strategy and their utilities are 0 and efficiency is low. In other diverse populations, they have different properties. In global adaptation, efficiency is moderate and equity is high. In local adaptation to random neighbors, both efficiency and equity are low. With the heterogeneous neighbors, both efficiency and equity become high.

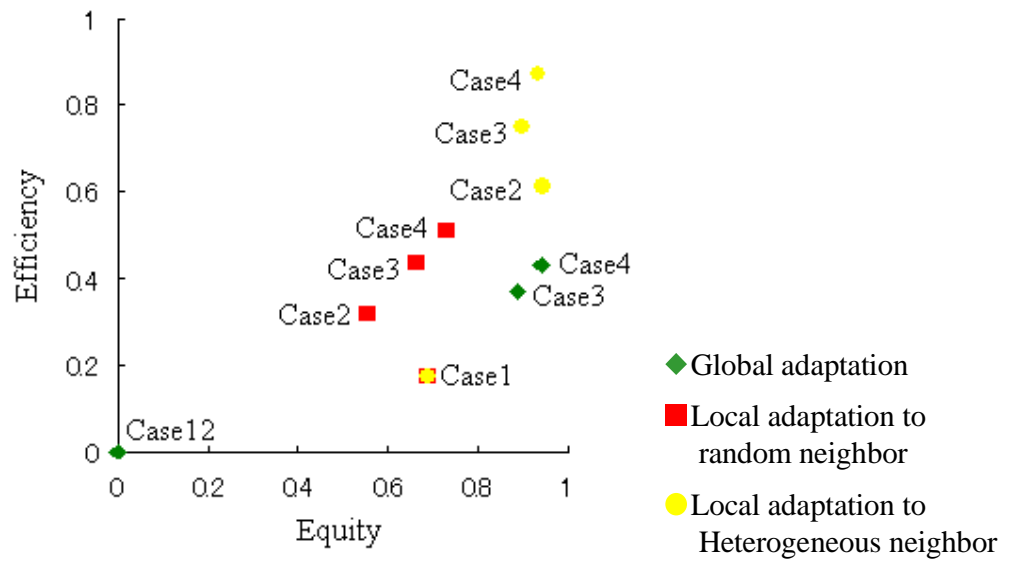

\section{Conclusion}

Fig.16: Efficiency and Equity (the initial proportion: 0.5)

In this paper we addressed the issue of collective decisions by heterogeneous agents in which they have to realize both efficient and equitable utilization of limited resources. Agents normally react to aggregate of others' decisions, and the resulting volatile collective decision is often far from being efficient. By means of experiments, we showed that the overall performance depends crucially on the types of interacting decisions as well as the heterogeneity of agents in term of their preferences. We considered two different types of interaction, symmetric and asymmetric interaction. We showed that the most crucial factor that 
considerably improves the overall of the system is the way of information presentation to agents on aggregates. It was shown that the global information presentation on the whole aggregate is inefficient. The optimal guidance strategy to improves the efficiency and the fairness depends on the way of interactions. With symmetric coordination, the local information presentation regarding neighbor of the same type realizes the highest performance. With asymmetric coordination, however, the local information regarding neighbor of the different type realizes the highest performance.

\section{References}

[1] Arthur W.B., "Inductive reasoning and Bounded Rationality", American Economic Review, vol.84, pp.406-411, 1994.

[2] Bakhchandani S., Hirshleifer D. and Welch I., "A Theory of Fad, Fashion, Custom, and Cultural Change as Informational Cascades". Journal of Political Economy, vo.100, no.5, pp.992-1026, 992.

[3] Bala V. and Goyal S., "Self-Organization in Communication Networks", Econometrica, vol.68, pp.1181-1230, 2000.

[4] Bala V. and Goyal S., "Learning from neighbours", Review of Economic Studies, vol.65, pp.595-621, 1998.

[5] Bala V. and Goyal S., "A non-cooperative model of network formation", Econometrica, vol. 68, pp.1181-1229, 2000.

[6] Banerjee. S., Efficiency and Stability in Economic Networks, mimeo: Boston University, 1999.

[7] Challet D. and Zhang C., "Emergence of Cooperation and Organization in an Evolutionary Game", Physica, A246, 1997.

[8] Durlauf S.N. and Young H.P., Social Dynamics, Brookings Institution Press, 2001.

[9] Dutta B. and Jackson M.O., "The Stability and Efficiency of Directed Communication Networks", Review of Economic Design, vol.5, p.251-272, 2000.

[10] Dutta B. and Jackson M.O., Introductory chapter in Models of Formation of Network Groups, Springer-Verlag, 2001.

[11] Ellison G., "Learning Local interaction, and Coordination", Econometrica, vol.61, pp.1047-1071, 1993.

[12] Fudenberg, D., and Levine, D., The Theory of Learning in Games, The MIT Press, 1998.

[13] Granovetter, M., "Threshold models of collective behavior" American Journal of Sociology, vol.183, pp.1420-1433, 1978.

[14] Harsarnyi, J. and Selten, R., A Game Theory of Equilibrium Selection in Games, MIT Press, 1988.

[15] Helbing D., Quantitative Sociodynamics, Kluwer Academic, 1995.

[16] Hofbauer, J., Sigmund, K., Evolutionary Games and Population Dynamics, Cambridge Univ. Press, 1998.

[17] Huberman B., Glance N., "Diversity and collective Action", Interdisciplinary Approaches to Nonlinear Systems, Springer, 1993.

[18] Iwanaga S. and Namatame. A., "Asymmetric Coordination of Heterogeneous Agents", IEICE Trans. on Information and Systems, vol. E84-D, pp.937-944, 2001.

[19] Iwanaga S. and Namatame. A., "The Complexity of Collective Decision", Journal of Nonlinear Dynamics and Control, vol.6, no.2, pp.137-158, 2002.

[20] Johnson C. and Gilles R. P., "Spatial Social Networks", Review of Economic Design, col.5, pp.273-300, 1999.

[21] Kaniovski, Y., Kryazhimskii, A., \& Young, H. Adaptive Dynamics in Games Played by Heterogeneous Populations. Games and Economics Behavior, 31, 50-96,2000.

[22] Kirman A., "The Economy as an Evolving Network", Journal of Evolutionary Economics, vol.7, pp. 339-353, 1997.

[23] Matteo M., "Toy Models of Markets with Heterogeneous Interacting Agents", Lecture Notes in Economics and Mathematical Systems, vol.503, pp.161-181, Springer, 2001.

[24] Nowak M. A., May R. M., "Evolutionary Games and spatial Chaos", Nature, 359, pp. 826-829, 1992.

[25] Rubinstein A., Modeling Bounded Rationality, The MIT Press, 1998.

[26] Schelling T., Micromotives and Macrobehavior, Norton, 1978.

[27] Weibull J., Evolutionary Game Theory, The MIT Press, 1996.

[28] Wolpert, D., Wheeler. K., and Tumer, K, "Collective Intelligence for Control of Distributed Dynamical Systems" Europhysics Letters, vol. 49, no. 6, 2000.

[29] Wolpert D. and Tumer K., "Optimal Payoff Functions for Members of Collective", In Advances in Complex Systems, 2001. 